\title{
Successful Diagnosis of Spinocerebellar Ataxia in a Patient Presenting with Progressive Incoordination of Movements
}

Roha Saeed Memon ${ }^{1 *}$, Iqra Saeed Memon ${ }^{2}$ and Muhammad Saeed Memon ${ }^{1}$

${ }^{1}$ Dow University of Health Sciences (DUHS), Karachi, Pakistan

${ }^{2}$ Liaquat University Hospital, Pakistan

"Corresponding author: Roha Saeed Memon, Medical Student, Dow University of Health Sciences (DUHS), Karachi, Pakistan, Tel: 922199215754; E-mail: memon.roha@gmail.com

Received date: January 12, 2017; Accepted date: January 23, 2017; Published date: January 25, 2017

Copyright: (c) 2017 Memon RS, et al. This is an open-access article distributed under the terms of the Creative Commons Attribution License, which permits unrestricted use, distribution, and reproduction in any medium, provided the original author and source are credited.

\begin{abstract}
A number of disorders cause ataxia which are the determinants of the choice of treatment for the patients affected by it. Correct diagnosis of the underlying disorder depends, not only on the qualification of the attending doctor but also on the accurate communication of the patient with the doctor. History thus plays a vital role in the diagnosis. Proper management might follow counselling sessions at times just to highlight the importance of history to a patient who is unaware about it. Here we present the case of a 32-year-old male who was experiencing progressive motor impairment. This report highlights the value of proper history in the diagnosis of the disease our patient is affected with.
\end{abstract}

Keywords: Spinocerebellar Ataxia; Neurodegenerative disease; Cerebellar atrophy

\section{Introduction}

Ataxia, which describes a lack of muscle coordination, is a nonspecific clinical manifestation implying dysfunction of those parts of the nervous system that coordinate movements such as the cerebellum and thus, points out to some underlying disease which as in our patient was diagnosed to be Spinocerebellar ataxia.

Spinocerebellar ataxia (SCA), of which there are several types, is a neurodegenerative disease which involves the cerebellum and its connections [1]. It is an autosomal dominant hereditary disease characterized by incoordination and loss of balance of motor functions which is often associated with poor coordination of hands, speech and eye movements which is slowly progressive and fatal [2].

In the cerebellum, the dysfunction and death of Purkinje cells, granule cells or interneurons can cause SCA [3]. Molecular mechanisms for this pathology commonly include polyglutamine tract expansion [4], flawed basal transcription and defective DNA repair.

There are approximately 60 types of SCA although not all the types cause severe equal disability. An estimated 150,000 people in the USA have a diagnosis of spinocerebellar ataxia at any given time. In UK, however, 1-3 out 100000 people are reportedly affected. It can affect anyone of any age.

This disease, being irreversible has no cure. However, the quality of life can be improved by controlling the symptoms associated with the sub-type, the patient is affected with. Adaptive devices such as canes or walkers may help a person to maintain their independence. Occupational, speech, or physical therapy might also help.

Here we present a patient with symptoms of progressive incoordination of voluntary movements. Ruling out possibilities via Laboratory test reports, detailed history and some typical presentations lead to the successful diagnosis of the underlying disease.

\section{Case History}

A 32-year-old male presented in a tertiary care hospital on 10th November, 2016 with complaints of difficulty in walking for four years. The attendant further tells us that he used to walk normally at first but then he felt like losing balance and fell forward. The episodes progressively increased in frequency and for the last year, patient has been almost bed bound. However, he still managed to get to the bathroom but with the support of two people. A week ago, he tried to walk independently but fell down hurting his knee. We were told that the patient had developed stiffness and some sort of deformity in his hands which he then couldn't work with at all. He was fully aware of what was being said to him and knew what to reply but was unable to utter words thus his speech was affected as well. The condition was diagnosed by the doctors as ataxia.

He had no history of alcohol intake or of any significant feud intake, no history of fever, diarrhea or weight loss.

Since the attendants refrained from disclosing the fact that this condition ran in their family earlier, the doctors concerned only on the non-genetic causes of ataxia and ordered several lab tests accordingly to rule out the possibilities and reach a conclusion. Thyroid test, Vitamin-B12 test, Blood CP, Blood sugar, all turned out to be normal. His cranial nerve examination was normal. MRI scan of the brain, being otherwise normal, showed significant cerebellar atrophy. The doctor also mentioned that the patient had exaggerated reflexes $(3+$ in all four limbs), droopy eyelids and tremulous tongue. This together with the cerebellar atrophy gave a slight hint towards spinocerebellar ataxia since these are some of the typical presentations of the disease and also, because non-genetic causes were ruled out based on the lab reports and examination. Since it is a genetic condition, Genetic diagnosis could've diagnosed it easily but since it is expensive and the technique is not available at most of the tertiary hospitals in Pakistan, an alternative method had to be used which in our case was the family history only. 
Citation: Memon RS, Memon IS, Memon MS (2017) Successful Diagnosis of Spinocerebellar Ataxia in a Patient Presenting with Progressive Incoordination of Movements. J Neurol Disord 5: 326. doi:10.4172/2329-6895.1000326

Page 2 of 3

On cross-questioning the attendants again, the doctors, however, found out about the fact that his family history was strongly positive indicating the disease to be a hereditary type. His mother and three out of six siblings had the same condition which together with the fact that there had been no inter-marriages in the family further implicated it to be an autosomal dominant type hereditary disease. This led to the successful diagnosis of the disease named spinocerebellar ataxia.

The patient and his attendants were counselled about the management of the disease and sent home.

\section{Discussion}

Cerebellum is the part of the brain responsible for balance and posture of the body by maintaining the coordination between various voluntary movements. Thus any condition that affects it adversely would result in loss of the normal functions carried out by it, the condition being named as ataxia. Ataxia itself is not a disease but a sign to some underlying disorder which should be diagnosed cautiously as the treatment depends mainly on the type of the disorder causing ataxia and not on ataxia itself.

There are two possible disease patterns which can lead to ataxia, genetic and non-genetic. Family history and genetic test results are the main distinguishing factors between the two. Genetic diagnosis being unavailable in our case, left us with family history alone which still was a powerful diagnosing factor. Negative history diverted our attention to the non-genetic causes and so several lab tests were ordered accordingly; Thyroid test, Vitamin-B12 test, Blood CP, Blood sugar, were all normal. His normal cranial nerve examination together with no history of weight loss ruled out posterior fossa tumors which was further confirmed by an MRI scan. MRI showed no evidence of ischemia or hemorrhage or any sclerotic plaque. It however showed Cerebellum atrophy which explained the loss of balance and incoordination of movements (Figure 1).
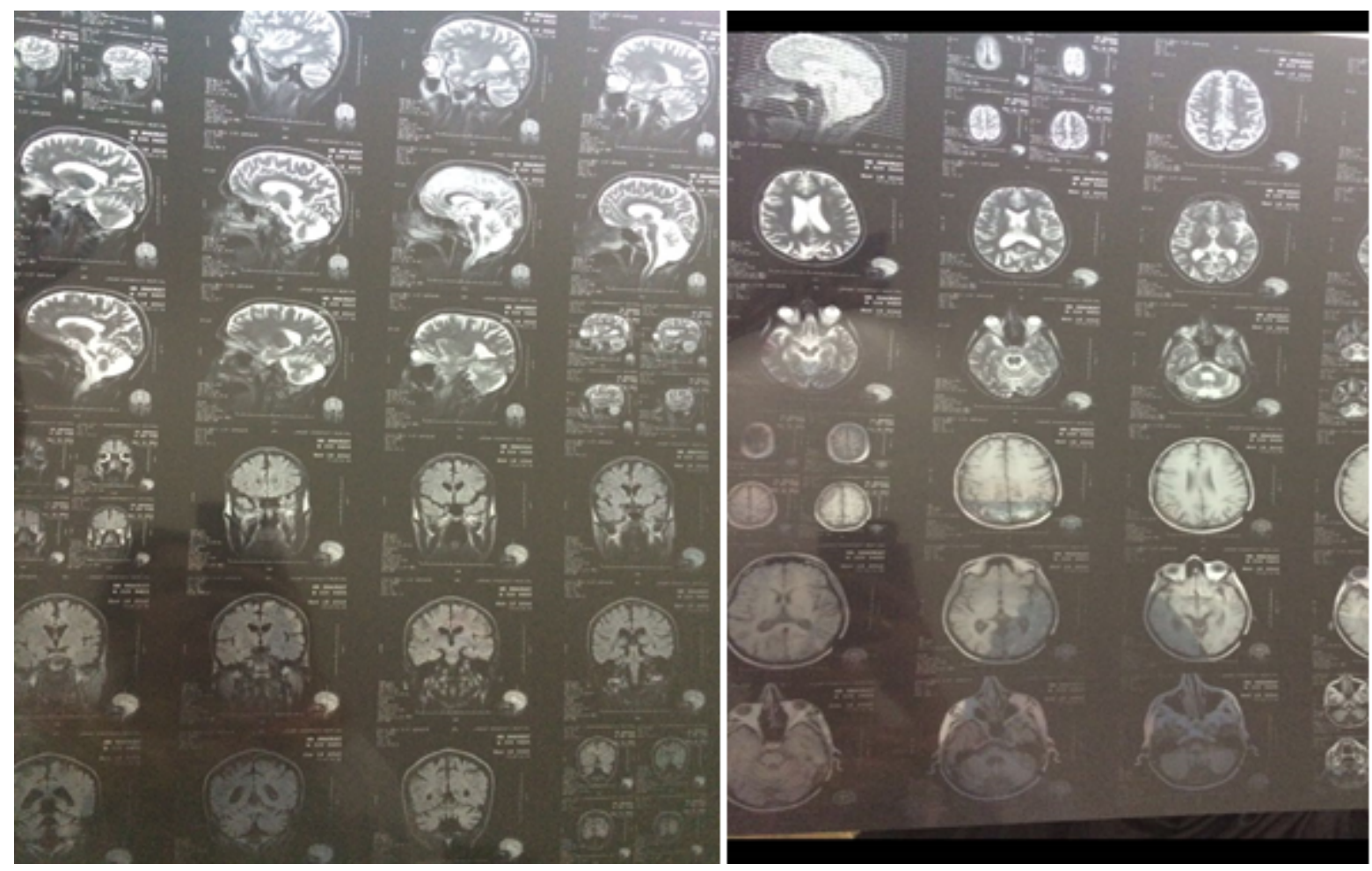

Figure 1: MRI scans showing cerebellar atrophy.

Based on the results, the case thus took a sharp turn away from all the other possible disease pathways but genetic. A detailed history was taken again but this time with the attendants being counseled along with as to what importance family history holds in the diagnosis of our case. We were then told that this condition does run in the family and so the disease is of a genetic origin. This proved to be a milestone as it led to the successful diagnosis of the disease. The classic pattern of inheritance i.e., a parent and $50 \%$ of his siblings affected by the same condition with no reported inter-marriages in the family, then distinguished this disease from an autosomal recessive condition, an example of which is Huntington's disease to be an autosomal dominant one. An autosomal dominant disease pattern presenting typically with ataxia, exaggerated reflexes, drooped eyelids and significant atrophy of the cerebellum led to the successful diagnosis of spinocerebellar ataxia.

With this, started another counselling session. This included informing all the concerned people about the fact that this disease has no cure yet and the life expectancy of the patient has significantly shortened due to the damage caused to one of the vital organs. However, the symptoms can be controlled and the quality of life improved which was thus followed by detailed guidance regarding the management of the disease. 
Citation: Memon RS, Memon IS, Memon MS (2017) Successful Diagnosis of Spinocerebellar Ataxia in a Patient Presenting with Progressive Incoordination of Movements. J Neurol Disord 5: 326. doi:10.4172/2329-6895.1000326

Page 3 of 3

\section{Conclusion}

Correct visualization of the presenting complains, an accurate history and examination, related lab test reports and proper knowledge all form a significant part of the diagnosis. With most of these factors related mainly to the capability of the attending doctor and the hospital concerned, history is the only part which has an equal role played by the doctor and the patient/attendant as we saw in our case. Had we been told about the positive family history and that too with a classic autosomal dominant pattern, the diagnosis would've been comparatively quicker and easier. Thus, history at times might need counselling to precede it as if the patient or his attendant is not fully conscious of the role he plays by virtue of giving a correct history and the role that information has in his treatment, can be dangerous and even fatal for his life.

\section{References}

1. U.S. National Library of Medicine (1998) Spinocerebellar Ataxia. Genes and Disease [Internet]: National Center for Biotechnology Information, Bethesda MD USA.

2. Aizawa CY, Pedroso JL, Braga-Neto P, Callegari MR, Barsottini OG (2013) Patients with autosomal dominant spinocerebellar ataxia have more risk of falls, important balance impairment, and decreased ability to function. Arg Neuropsiquiatr 71: 508-511.

3. Obayashi M, Synofzik M, Stevanin G, Monin ML, Duyckaerts C, et al. (2015) Spinocerebellar ataxia type 36 exists in diverse populations and can be caused by a short hexanucleotide GGCCTG repeat expansion. J Neurol Neurosurg Psychiatry 86: 986-995.

4. Durr A (2010) Autosomal dominant cerebellar ataxias: polyglutamine expansions and beyond. Lancet Neurol 9: 885-894. 